\title{
Probabilistic Approach to Predict Abnormal Combustion in Spark Ignition Engines
}

\author{
Mohammed Jaasim Mubarak Ali, Minh Bau Luong, Aliou Sow, Francisco Hernandez Perez, and Hong Im \\ King Abdullah University of Science \& Technology (KAUST)
}

\author{
Citation: Mubarak Ali, M.B. Luong, A. Sow, F.H. Perez, H. Im, "Probabilistic Approach to Predict Abnormal Combustion in Spark Ignition \\ Engines," SAE Technical Paper 2018-01-1722, 2018, doi:10.4271/2018-01-1722.
}

\begin{abstract}
This study presents a computational framework to predict the outcome of combustion process based on a given RANS initial condition by performing statistical analysis of Sankaran number, Sa, and ignition regime theory proposed by Im et al. [1]. A criterion to predict strong auto-ignition/detonation a priori is used in this study, which is based on Sankaran-Zeldovich criterion. In the context of detonation, $\mathrm{Sa}$ is normalized by a sound speed, and is spatially calculated for the bulk mixture with temperature and equivalence ratio stratifications. The initial conditions from previous pre-ignition simulations were used to compute the spatial Sa distribution followed by the statistics of Sa including the mean $\mathrm{Sa}$, the probability density function (PDF) of Sa, and the detonation probability, $\mathrm{P}_{\mathrm{D}}$. Sa is found to be decreased and detonation probability increased significantly with increase of temperature. The statistic mean Sa calculated for the entire computational domain and the predicted Sa from the theory were found to be nearly identical. The predictions based on the adapted Sankaran-Zel'dovich criterion and detonation probability agree well with the results of the previous high fidelity pre-ignition simulations.
\end{abstract}

\section{Introduction}

A downsized spark ignition (SI) engine is capable of reducing pollutant emissions and operate at high efficiencies, but is limited by the problem of pre-ignition that may result in super-knock [2-4]. Engines are operated at boosted intake pressure to account for the power loss due to downsizing of the engine [5]. Pre-ignition refers to a premature ignition of the air/fuel mixture before spark firing and through an unknown source, and the subsequent event that is characterized by high pressure peaks and oscillations is called superknock [6]. Such an event can severely damage the moving components of the engine such as valves, piston and piston rings resulting in loss of the engine [7]. Auto-ignition of hot-spots followed by detonation is reported as the predominant reason for super-knock [8].

Our previous study [9] emphasized the importance of the temperature, mixture stratification, and low-temperature reactivity in end-gas on super-knock. The location of the hotspot that initiates preignition front is found to be of paramount importance in determining the intensity of super-knock. Early pre-ignition consumes the air/fuel mixture completely with no super-knock event. Late pre-ignition near top dead center resulted in super-knock with severe pressure oscillations. The increased temperature of the end-gas due to the preignition front and low-temperature reactivity in the end-gas were Page 1 of 7 found to be major factors favoring the occurrence of super-knock.

The in-cylinder hot-spots are responsible for the pre-ignition and subsequent detonation that leads to super-knock $[8,10]$. In a recent study by the authors [11], it was demonstrated that during preignition, the hot-spots in the stratified mixture undergoes autoignition followed by detonation causing super-knock. In another scenario, the pre-ignition front triggered detonation of the end gas pocket at close proximity to hotspot.

The source that a causes pre-ignition is not known in experiments and multiple sources are reported in literature that is responsible for preignition, such as oil droplets, carbon deposits, hot surfaces etc., [4, 12-16]. However, it is important to realize that whether the preignition leads to detonation/super-knock in the end gas depends strongly on the property of the bulk gas, such as temperature and composition distribution. To this end, the main objective of this study is to propose a methodology to predict strong autoignition/detonation that leads to super-knock based on the SankaranZel'dovich criterion [17] and validate the approach with previously reported simulations [11]. Detonation probability is calculated to obtain quantified results and validate the proposed probabilistic approach.

\section{Computational Setup (3-D)}

A short description of the modeling methodology used to obtain the simulation results is presented. Three dimensional (3-D) computational fluid dynamics (CFD) full cycle simulations were performed using CFD code, CONVERGE, to obtain the initial temperature and mixture stratification for the study. A grid size of $0.125 \mathrm{~mm}$ is used in all directions with $\sim 0.5$ million cells at top dead center. The in-cylinder turbulent flow is described by large eddy simulations using the dynamic structure model [18]. The heat loss model of Han et al. [19] is used to account for wall heat losses, which are of paramount importance, causing temperature stratification inside the cylinder. The engine specifications and simulation conditions adapted for the study are provided in Table 1. More details about the 3-D setup can be found in [11].

\section{Numerical Setup (2-D)}

The results from 3-D full cycle SI engine simulations were used to obtain the initial conditions for 2-D pre-ignition simulations. Temperature, species mass fractions and velocity distributions were obtained at TDC ( $8 \mathrm{~mm}$ from spark plug) and mapped onto a 2-D 
circular domain of $85 \mathrm{~mm}$ diameter equal to the size of cylinder bore as shown in Figure 1. A minimum cell size of $25 \mu \mathrm{m}$ is used for all the simulated cases. A deflagration front initiated at the symmetric axis near the end-wall as shown in Figure 2(a). The multi-zone SAGE detailed chemistry solver, based on well-stirred reactor assumption, is used to solve for chemical kinetics [20]. The initial temperature, 700 $\mathrm{K}$ is increased to $1280 \mathrm{~K}$ at different temperature increments (refer Table 2) to elucidate the relationship between hotspots, gradients of temperature, mixture fraction distribution over strong autoignition/detonation over super-knock. Table 2 shows the initial conditions for the simulations and the observed high fidelity preignition results of the cases selected for the study. More details about the case setup, grid independence of the solution, detailed numerical results and modeling methodology adopted for the study can be found in [11].

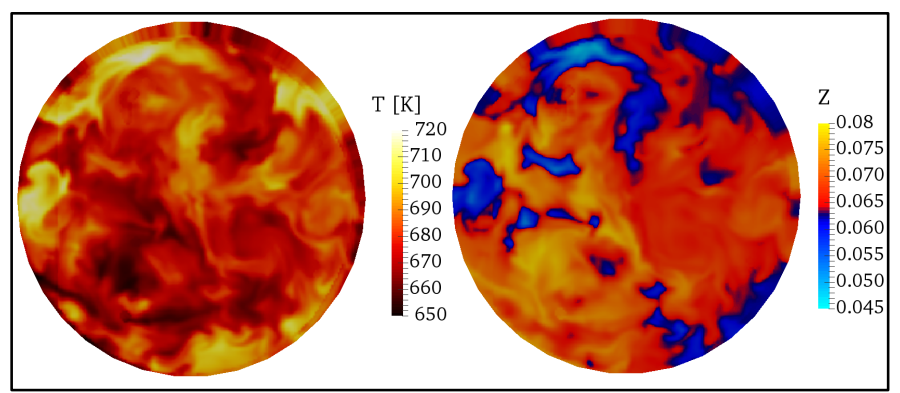

\section{Pre-ignition to Super-knock Transition}

The 2-D simulations reproduced the entire event: an imposed hotspot initiated deflagrative front which in turn transformed into detonation front leading to super-knock, consistent with direct numerical studies by Sow et al. [21]. The detonation front is accompanied with local auto-ignitions in the end gas, at locations of hot-spots due to increased reactivity as shown in Figure 2(d) for case $2(T=1080 \mathrm{~K})$. While not shown here, similar trend is observed for other cases where temperature is greater than $800 \mathrm{~K}$. The results of all simulation cases with different temperatures are summarized in Table 2. The readers may refer to [11] for detailed results of the cases mentioned in Table 2. The local peaks in pressure plot (Figure 2 (inset)) indicates the flame initiation, other peaks corresponds to detonation initiation (Figure $2(\mathrm{~b}, \mathrm{c})$ ), auto-ignitions (Figure $2(\mathrm{~d}, \mathrm{e})$ ) and auto-ignition front $\&$ detonation front interaction (Figure 2 (f)) at different time instants. The auto-ignition front undergoes detonation after initiation, causing local sharp peak pressures. The magnitude of pressure peaks increases towards end of combustion due to multiple auto-ignitions that transition into detonation in the end gas, which indicates super-knock. The results also demonstrate the capability of the adapted modeling methodology to simulate pre-ignition and super-knock.

Figure 1. Initial temperature and mixture fraction distribution mapped onto a 2-D domain.

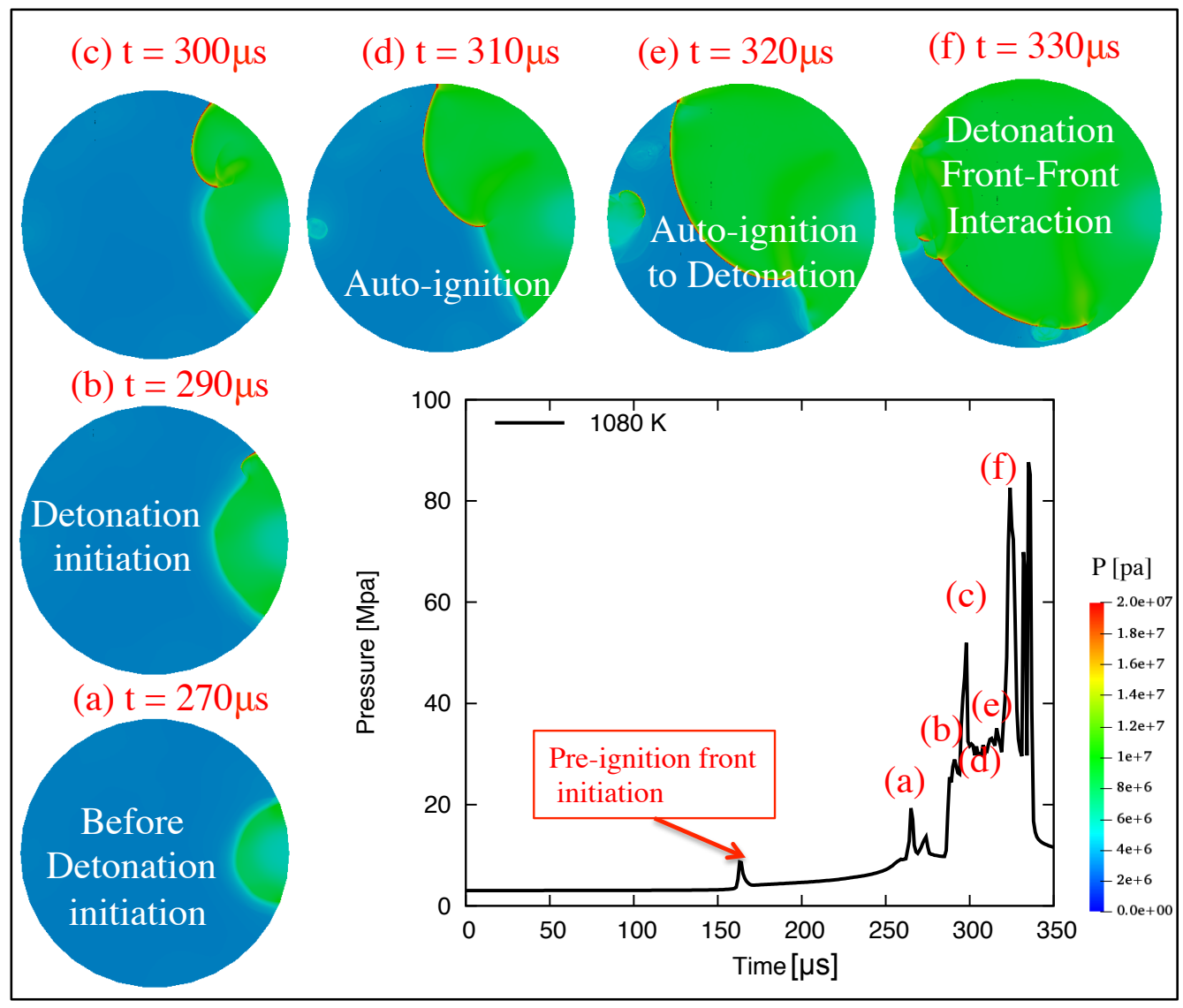

Figure 2. Pressure contour showing detonation initiation and auto-ignition at various times for case $2(\mathrm{~T}=1080 \mathrm{~K}$

Page 2 of 7 
Table 1. Engine specifications and operating conditions for the study.

\begin{tabular}{|l|l|}
\hline Specifications & Values \\
\hline Stroke & $90 \mathrm{~mm}$ \\
\hline Bore & $85 \mathrm{~mm}$ \\
\hline Connecting rod & $138 \mathrm{~mm}$ \\
\hline Compression ratio & 9.5 \\
\hline Number of valves & 4 \\
\hline Spark plug energy & $40 \mathrm{~mJ}$ \\
\hline Operating conditions & Values \\
\hline RPM & 1200 \\
\hline Intake temperature & $300 \mathrm{~K}$ \\
\hline Intake pressure & 2 bar \\
\hline Global equivalence ratio & 1 \\
\hline Fuel & iso-octane \\
\hline Spark timing & 5 CAD aTDC \\
\hline
\end{tabular}

Table 2.Initial temperature for the parametric study and their outcomes [11].

\begin{tabular}{|l|l|l|}
\hline Case No & Initial Temperature (K) & Observation \\
\hline 1 & 700 & Deflagration \\
\hline 2 & $800($ NTC) & Slow detonation at EOC \\
\hline 3 & $850($ NTC) & Slow detonation at EOC \\
\hline 4 & $900($ NTC) & Slow detonation at EOC \\
\hline 5 & 950 & Slow detonation at EOC \\
\hline 6 & 1000 & Slow detonation at EOC \\
\hline 7 & 1080 & Fast detonation at SOC \\
\hline 8 & 1280 & Fast detonation at SOC \\
\hline
\end{tabular}

SOC-Start of combustion, EOC-End of Combustion, NTC-Negative Temperature Coefficient Regime

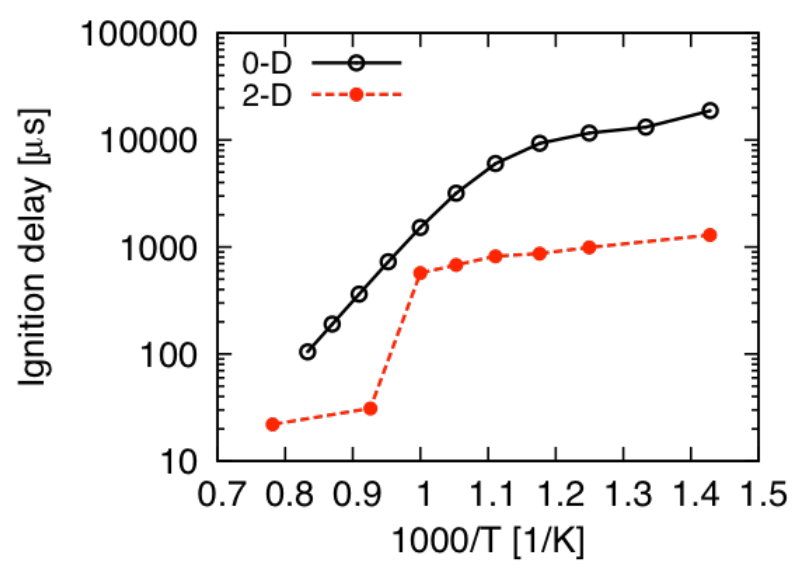

Figure 3. Comparison of ignition delay between 0-D and 2-D simulations.

\section{Calculation of Detonation Probability - A Predictive Method}

Based on the results in the previous sections, pre-ignition front transitions into detonation leading to super-knock (see Figure 2).

Page 3 of 7
Therefore, a scaling analysis based on modified Zel'dovich criterion proposed by Sankaran et al. [17] is used to predict the probability of detonation a priori, and validate the results with the previous simulation results in [11]. First, to calculate detonation probability from initial conditions, the non-dimensional Sankaran number, $\mathrm{Sa}$, is adopted, which is based on the initial field of temperature and mixture gradients to predict the outcome of the simulations [17]. Sankaran number is defined as

$$
\begin{aligned}
& S a=\beta \frac{S_{l}}{S_{s p}} \\
& S_{s p}=\left|\nabla \tau_{i g}\right|^{-1}
\end{aligned}
$$

where $S_{1}$ is the laminar flame speed, $S_{s p}$ is the spontaneous front propagation speed, and $\beta=0.5$ is a weighted factor proposed by Sankaran et al. [17]. The value of $\beta$ is chosen to lower than unity based on assumption that there is a delay in deflagration front initiation in spite of mixture being ripe for deflagration front initiation from an ignition kernel. It is reported that results were found to be consistent even with lower values of $\beta$ [17]. The above value of $\beta$ was proposed for cases where ignition kernel transitions to deflagration front, whereas in the present study $\beta$ is approximately equal to be the ratio of the ignition delay of 2-D simulations to the homogeneous 0 -D ignition delay. The mean value of above ratio 0.1 is used for $\beta$ in the present study. Comparison of the ignition delay values between 0-D and 2-D is shown in Figure 3. The ignition delay of $2-\mathrm{D}$ simulations is defined as the time at the $50 \%$ cumulative heat release.

By definition, the Zeldovich-Sankaran criterion predicts a weak ignition if $\mathrm{Sa}>1$ in which the deflagration front dominates the ignition process; whereas a strong ignition or spontaneous ignition dominates if $\mathrm{Sa}<1$. To predict the possibility of detonation, $\mathrm{Sa}$ number is evaluated based on sound speed, $a$, rather than laminar flame speed, $\mathrm{S}_{l}$, such that

$$
S a=\beta \frac{a}{S_{s p}}
$$

With temperature fluctuation only, $S_{s p}$ is typically expressed as

$$
S_{s p}=\left|\frac{\partial \tau_{i g}}{\partial T} \nabla \tau_{i g}\right|^{-1}
$$

where $\tau_{i g}$ is the ignition delay time for a homogeneous mixture, $\partial T / \partial \tau_{i g}$ represents the ignition delay sensitivity to temperature, and $\nabla T$ is the initial temperature gradient as in $[1,22]$. For a fuel/air mixture with both temperature and equivalence ratio fluctuations, $S_{s p}$ is expressed as

$$
S_{s p}=\left|\frac{\partial \tau_{i g}}{\partial T} \nabla T+\frac{\partial \tau_{i g}}{\partial \phi} \nabla \phi\right|^{-1}
$$


Table 3. Initial mean temperature, $T_{0}$, ignition delay, $\tau_{\mathrm{ig}}$, speed of sound, $a$, integral scale Damköhler number, $D \mathrm{a}_{1}$, partial ignition delay gradients with respect to temperature and equivalence ratio, $\partial \tau_{i} / \partial T$ and $\partial \tau_{\mathrm{ig}} / \partial \phi, K$ factor, predicted Sankaran number, predicted Sa, mean Sankaran number, mean Sa, detonation probability, $P_{\mathrm{D}}$ (Sa normalized by sound speed), probability of strong ignition, $P \mathrm{~s}$ (Sa normalized by laminar flame speed). From statistical analysis, the root mean square of temperature and equivalence ratio of $T^{\prime}=12 \mathrm{~K}$ and $\phi^{\prime}=0.1$, respectively, are calculated from initial conditions. The root mean square of velocity and the integral length scale are $u^{\prime}=0.82 \mathrm{~m} / \mathrm{s}$ and $l_{\mathrm{e}}=1.57 \mathrm{~mm}$, and the corresponding turbulent time scale, $\tau_{\mathrm{t}}$, is $1.9 \mathrm{~ms}$.

\begin{tabular}{|c|c|c|c|c|c|c|c|c|c|c|c|}
\hline Case & $T_{0}$ & $\tau_{i g}$ & $a$ & $D a_{l}$ & $\frac{\partial \tau_{i g}}{\partial T}$ & $\frac{\partial \tau_{i g}}{\partial \phi}$ & $K$ & $\begin{array}{c}\text { Predicted } \\
\mathrm{Sa}\end{array}$ & $\begin{array}{c}\text { Mean } \\
\mathrm{Sa}\end{array}$ & $P_{D}$ & $P_{S}$ \\
\hline 1 & 700 & 18.8 & 680.0 & 0.10 & $-2.0 \mathrm{E}-1$ & $-5.5 \mathrm{E}+0$ & 22.40 & 70.1 & 61.6 & $2.1 \mathrm{E}-3$ & 0.92 \\
\hline 2 & 800 & 11.6 & 673.4 & 0.17 & $3.4 \mathrm{E}-3$ & $-5.4 \mathrm{E}+0$ & 6.93 & 17.0 & 16.3 & $3.4 \mathrm{E}-2$ & 0.99 \\
\hline 3 & 850 & 9.28 & 637.7 & 0.21 & $-1.9 \mathrm{E}-2$ & $-4.2 \mathrm{E}+0$ & 2.03 & 4.5 & 9.5 & $4.3 \mathrm{E}-2$ & 0.99 \\
\hline 4 & 900 & 6.01 & 636.6 & 0.32 & $-3.9 \mathrm{E}-2$ & $-2.4 \mathrm{E}+0$ & 4.04 & 7.1 & 10.5 & $2.5 \mathrm{E}-2$ & 0.99 \\
\hline 5 & 950 & 3.18 & 647.1 & 0.60 & $-3.1 \mathrm{E}-2$ & $-1.1 \mathrm{E}+0$ & 5.90 & 7.6 & 8.8 & $3.6 \mathrm{E}-2$ & 0.99 \\
\hline 6 & 1000 & 1.52 & 632.7 & 1.26 & $-1.7 \mathrm{E}-2$ & $-5.0 \mathrm{E}-1$ & 4.59 & 4.1 & 4.7 & $1.0 \mathrm{E}-1$ & 0.99 \\
\hline 7 & 1080 & 0.35 & 643.5 & 5.52 & $-4.9 \mathrm{E}-3$ & $-1.3 \mathrm{E}-1$ & 2.76 & 1.2 & 1.4 & $4.9 \mathrm{E}-1$ & 1.0 \\
\hline 8 & 1280 & 0.03 & 650.5 & 58.52 & $-3.0 \mathrm{E}-4$ & $-6.6 \mathrm{E}-3$ & 0.51 & 0.1 & 0.1 & $1.0 \mathrm{E}+0$ & 1.0 \\
\hline
\end{tabular}

In the context of RANS, $\mathrm{S}_{s p}$ is approximated as

$$
S_{s p}=\left|\frac{\partial \tau_{i g}}{\partial T}\right|_{\phi_{0}} \nabla \tilde{T}+\left.\left.\frac{\partial \tau_{i g}}{\partial \phi}\right|_{T_{0}} \nabla \tilde{\phi}\right|^{-1}
$$

For a given initial field of temperature and equivalence ratio, $\mathrm{Sa}$ number is spatially computed based on the equations (3) and (6). For RANS simulations, Sa numbers are computed spatially at each cell throughout the entire computational domain such that statistical analysis of Sa distribution such as the mean $\mathrm{Sa}$, the $\mathrm{PDF}(\mathrm{Sa})$, and the volumetric fraction of $\mathrm{Sa}$ number less than 1.0 is performed. The probability density function (PDF) of Sa number for all the cases of different initial temperatures $(700 \mathrm{~K}-1280 \mathrm{~K})$ is shown in Figure 4.

The detonation probability $\left(P_{\mathrm{D}}\right)$ is defined as

$$
P_{D}=\frac{\text { Volume }_{(S a \leq 1)}}{\text { Volume }_{\text {Total }}}
$$

where Sa is evaluated based on the sound speed, $a$. The detonation probability $\left(\mathrm{P}_{\mathrm{D}}\right)$ is shown in Table 3 for all the cases. For reference, probability of normal combustion (Ps) in which $\mathrm{Sa}$ number is normalized by the laminar flame speed is also calculated and shown in Table 3 and plotted Figure 4(a). As seen in Figure 4(a), Sa is primarily lower than unity for all the cases except for case 1 with temperature of $700 \mathrm{~K}$. Therefore, it is more likely that the combustion of cases 2-8 occur in strong ignition/detonation. As seen in Figure 4(b) and visually shown in Figure 5, as temperature increases the $\operatorname{PDF}(\mathrm{Sa})$ becomes mostly lower less than unity for cases 7 and 8 , at temperature of $1080 \mathrm{~K}$ and $1280 \mathrm{~K}$, respectively. This implies that increasing temperature results in higher possibility of detonations, which is also confirmed by $P_{\mathrm{D}}$ in Table 3 and Figure $4(\mathrm{~b}) . P_{\mathrm{D}}$ exponentially decreases with increasing temperature. For example, case 8 of $1280 \mathrm{~K}$ with $P_{\mathrm{D}}=1$ is more likely to result in a strong detonation mode. On the contrary, detonation is not expected to occur for case 1 of $700 \mathrm{~K}$ with $P_{\mathrm{D}}=0.0021$. This statistical analysis is consistent with the observations from the previous pre-ignition
RANS results (refer to Table 2). In previous studies, strong and weak ignitions were well predicted by Sa number normalized by laminar flame speed $[1,23]$ with thermal stratification only. In this study, the prediction for detonation probability is made by the 'extended' $\mathrm{Sa}$ number normalized by sound speed, in the presence of both temperature and equivalence ratio fluctuations.
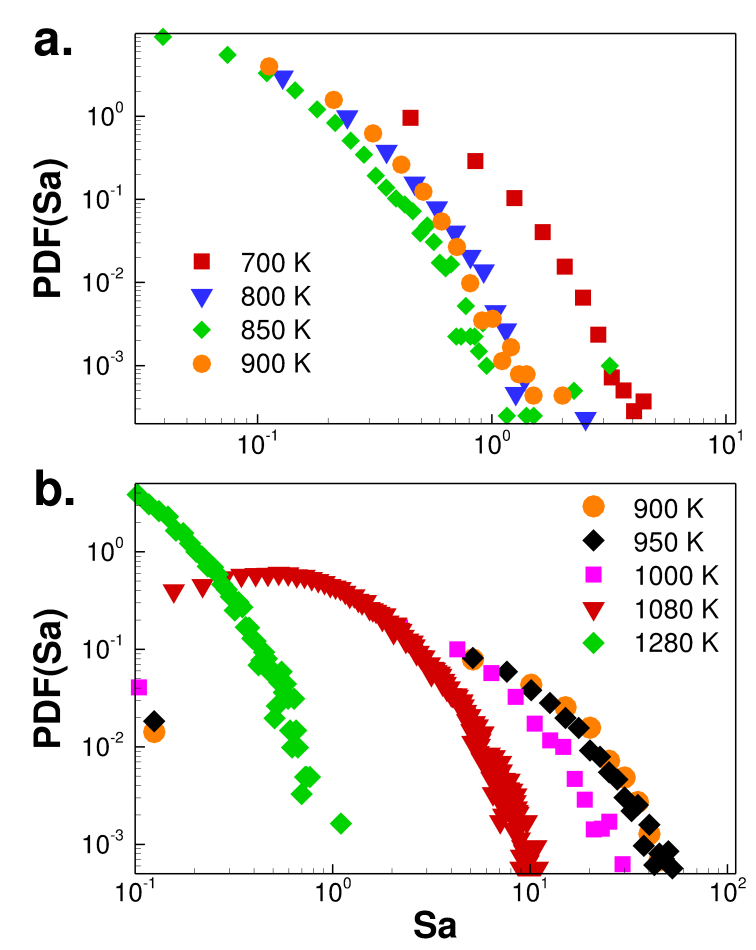

Figure 4. Probability density function of Sa number (a) normalized by laminar flame speed (see equation 1), (b) normalized by sound speed for different initial temperatures (cases 1-8). 


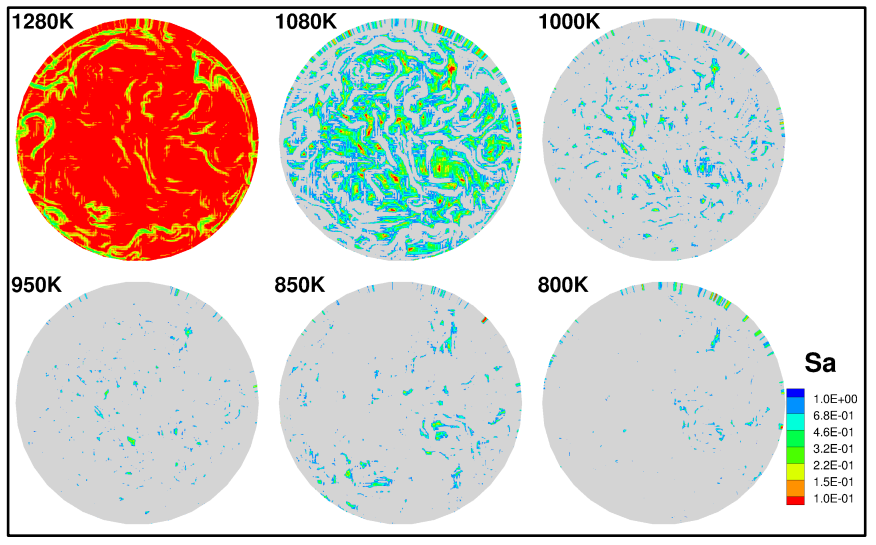

Figure 5. Isocontour of Sa number for different initial temperatures (cases 2$8)$.

Next, the predicted Sa number is compared with the mean Sa number, which is computed throughout the computational domain. According to Im et al. [1], Sa number is further simplified by performing the scaling analysis that results in a predicted Sa number defined as

$$
\begin{aligned}
& S a=K D a_{l}^{-1 / 2} \\
& K=\beta \frac{1}{\sqrt{\tau_{f} \tau_{i g}}}\left|\frac{d \tau_{i g}}{d T} T^{\prime}\right|
\end{aligned}
$$

In the presence of both temperature and equivalence ratio fluctuations and by assuming that the temperature and equivalence ratio fields have an identical integral length scale, the $K$ factor is written as

$$
K=\beta \frac{1}{\sqrt{\tau_{f} \tau_{i g}}}\left|\frac{\partial \tau_{i g}}{\partial T} T^{\prime}-\frac{\partial \tau_{i g}}{\partial \phi} \phi^{\prime}\right|
$$

where, $D \mathrm{a}_{l}$ is the Damköhler number, $T^{\prime}$ is the root mean square of temperature, $\phi^{\prime}$ is root mean square of equivalence ratio, $l_{\mathrm{e}}$ is the integral length scale, and $\tau_{f}$ is the flame time scale. The minus sign in equation (10) is attributed to the fact that temperature and equivalence ratio is nearly negatively-correlated due to the cooling effect of fuel vaporization, which in turn results in a negative correlation of the gradient of temperature and equivalence ratio as shown in Figure 6.

The predicted Sa is plotted as a function of temperature in Figure 7, which is nearly identical to the mean Sa number from the overall bulk mixture. The Sa number of case 8 (case 7) is much less than unity (close to unity) which again confirm that the combustion of case 8 and 7 have a high propensity to occur in detonation mode that is consistent with previous discussions. The results of this study demonstrate that by properly normalizing Sa number, it can be used to predict the outcome of the combustion process under various conditions including canonical DNS configurations [23] and RANS simulations, and in the experiments of rapid compression machines and engines [1].
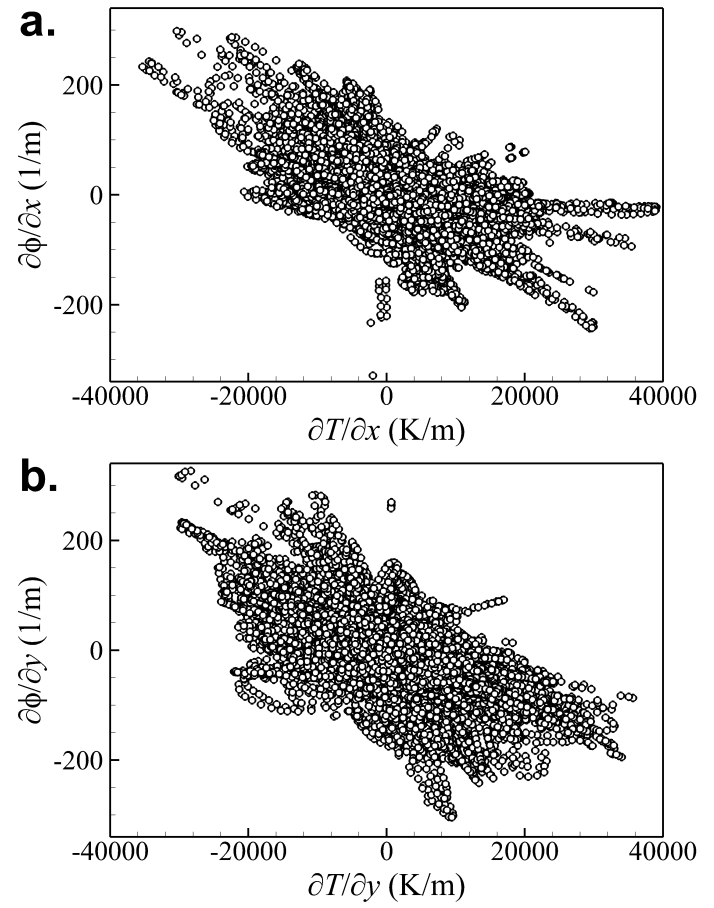

Figure 6. Relation between the gradient of temperature and the gradient of equivalence ratio in (a) $\mathrm{X}$-axis and (b) $\mathrm{Y}$-axis.
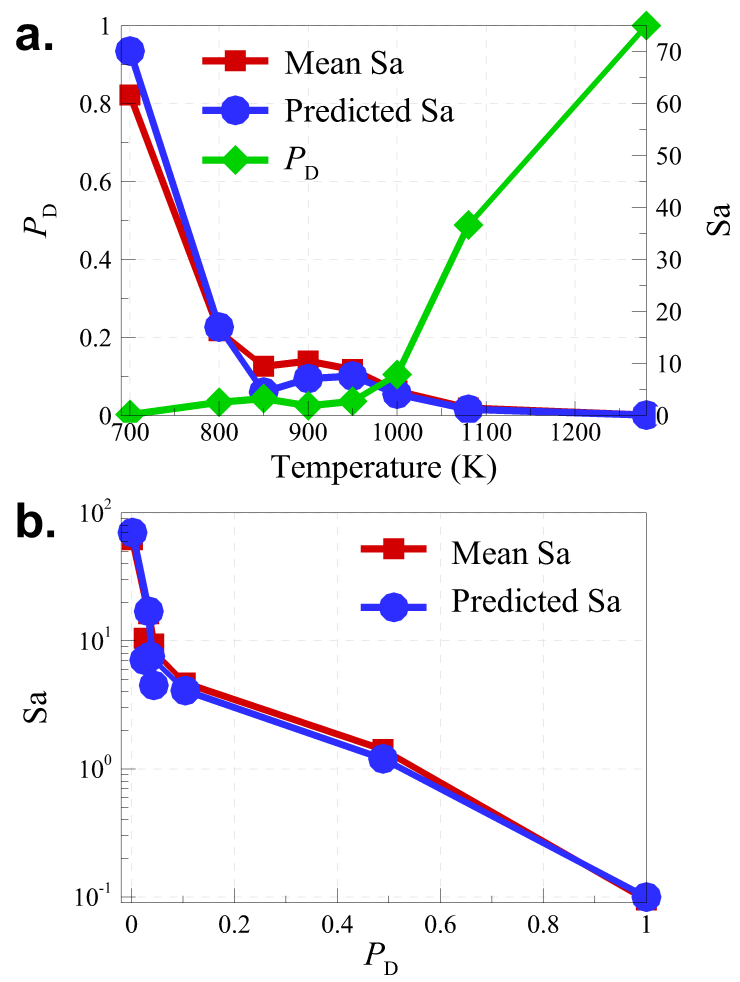

Figure 7. (a) Mean Sa number, predicted Sa number and detonation probability are plotted as a function of the initial mean temperature, (b) Mean Sa number and predicted Sa number plotted as function of detonation probability. 


\section{Conclusions}

In this study, we extend the previously developed theory of ignition regime diagram to apply for RANS context with both temperature and equivalence ratio stratifications under spark ignition engine conditions. A new non-dimensional Sa number is normalized by sound speed for prediction of detonation. The initial conditions from the previous RANS study are chosen to perform the statistical analysis of Sankaran number, Sa. The mean $\mathrm{Sa}, \mathrm{PDF}(\mathrm{Sa})$ and $P_{\mathrm{D}}$ are used to predict the livelihood of detonations and compare with previous RANS results. The predicted Sa number is found to be almost identical to the mean Sa number. It is also found that, as the initial mean temperature increases, the detonation probability, $P_{\mathrm{D}}$, significantly increase, resulting in a higher propensity of detonation occurrence. $P_{\mathrm{D}}$ and mean Sa number can be used as an indicator to predict the outcome of the combustion process. From this study, it can be concluded that the refined theory of ignition based on scaling analysis can be applied for various engine context from experiments to RANS-DNS studies to predict wide range of combustion modes of weak/strong ignitions as well as detonations.

The proposed detonation probability can be used as an indicator of super-knock in engines, which will be performed and validated further in future.

\section{References}

1. Im, H.G., Pal, P., Wooldridge, M.S., Mansfield, A.B., "A regime diagram for autoignition of homogeneous reactant mixtures with turbulent velocity and temperature fluctuations," Combustion Science and Technology, 187(8), 1263-1275, 2015, doi: 10.1080/00102202.2015.1034355.

2. Lecointe, B., Monnier, G., "Downsizing a gasoline engine using turbocharging with direct injection," SAE Technical Paper, 2003-010543, 2003, doi: 10.4271/2003-01-0542.

3. Leduc, P., Dubar, B., Ranini, A., Monnier, G., "Downsizing of gasoline engine: an efficient way to reduce $\mathrm{CO}_{2}$ emissions," Oil \& gas science and technology, 58(1), 115-127, 2003, doi: 10.2516/ogst:2003008.

4. Chapman, E.M., Costanzo, V.S., "A Literature Review of Abnormal Ignition by Fuel and Lubricant Derivatives," $S A E$ International Journal of Engines, 9(1), 107-142, 2015, doi:10.4271/2015-01-1869.

5. Dahnz, C., Han, K. M., Spicher, U., Magar, M. et al., "Investigations on pre-ignition in highly supercharged SI engines." SAE International Journal of Engines, 3(1), 214-224, 2010, doi:10.4271/2010-01-0355.

6. Kalghatgi, G., "Knock onset, knock intensity, superknock and preignition in spark ignition engines," International Journal of Engine Research, 19(1), 7-20, 2017 , doi: $10.1177 / 1468087417736430$.

7. Wang, Z., Liu, H., Song, T., Qi, Y., He, X., Shuai, S., Wang, J., "Relationship between super-knock and pre-ignition." International Journal of Engine Research, 16(2), 166-180, 2015, doi: 10.1177/1468087414530388.

8. Bates, L., Bradley, D., Paczko, G., Peters, N., "Engine hot spots: Modes of auto-ignition and reaction propagation." Combustion and Flame, 166, 80-85, 2016, doi: 10.1016/j.combustflame.2016.01.002. 9. Jaasim, M., Perez, F.E., Sivasankaralingam, V., Raman, V. et al., "Effect of Location and Timing of Hotspots on Superknock during Pre-ignition," SAE Technical Paper, 2017-01-0686, 2017, doi: 10.4271/2017-01-0686.

10. Bates, L., Bradley, D., "Deflagrative, auto-ignitive, and detonative propagation regimes in engines," Combustion and Flame, 175, 118-122, 2017, doi: 10.1016/j.combustflame.2016.05.023.

11. Jaasim, M., Sow, A., Hernandez Perez, F., Im, H.G., "A Computational Study of Abnormal Combustion Characteristics in Spark Ignition Engines, " SAE Technical Paper, 2018-01-0179, 2018, doi: $10.4271 / 2018-01-0179$.

Page 6 of 7
12. Gupta, A., Shao, H., Remias, J., Roos, J., et al., "Relative Impact of Chemical and Physical Properties of the Oil-Fuel Droplet on PreIgnition and Super-Knock in Turbocharged Gasoline Engines," SAE Technical Paper, 2016-01-2278, 2016, doi: 10.4271/2016-01-2278. 13. Mayer, M., Hofmann, P., Geringer, B., Williams, J. et al., "Influence of Different Oil Properties on Low-Speed Pre-Ignition in Turbocharged Direct Injection Spark Ignition Engines," SAE Technical Paper, 2016-01-0718, 2016, doi: 10.4271/2016-01-0718. 14. Qi, Y., Xu, Y., Wang, Z., Wang, J., "The effect of oil intrusion on super knock in gasoline engine," SAE Technical Paper, 2014-011224, 2014, doi: 10.4271/2014-01-1224.

15. Haenel, P., Kleeberg, H., de Bruijn, R., Tomazic, D., "Influence of Ethanol Blends on Low Speed Pre-Ignition in Turbocharged, Direct-Injection Gasoline Engines," SAE International Journal of Fuels and Lubricants, 10(1), 95-105, 2017,doi: 10.4271/2017-010687.

16. Haenel, P., Seyfried, P., Kleeberg, H., Tomazic, D., "Systematic approach to analyze and characterize pre-ignition events in turbocharged direct-injected gasoline engines," SAE Technical Paper, 2011-01-0343, 2011, doi: 10.4271/2011-01-0343.

17. Sankaran, R., Im, H.G., Hawkes, E.R., Chen, J.H., "The effects of non-uniform temperature distribution on the ignition of a lean homogeneous hydrogen-air mixture," Proceedings of the Combustion Institute, 30(1), 875-882, 2005, doi: 10.1016/j.proci.2004.08.176.

18. Chumakov, S.G., Rutland, C. J., "Dynamic structure subgridscale models for large eddy simulation," International journal for numerical methods in fluids, 47(8-9), 911-923, 2005, doi: 10.1002/fld.907.

19. Han, Z., Reitz, R.D., "A temperature wall function formulation for variable-density turbulent flows with application to engine convective heat transfer modeling," International journal of heat and mass transfer, 40(3), 613-625, 1997, doi: 10.1016/00179310(96)00117-2.

20. Babajimopoulos, A., Assanis, D., Flowers, D., Aceves, S. et al., "A fully coupled computational fluid dynamics and multi-zone model with detailed chemical kinetics for the simulation of premixed charge compression ignition engines," International journal of engine research, 6(5), 497-512, 2005, doi: 10.1243/146808705X30503.

21. Sow, A., Lee, B.J., Im, H.G., "A Computational Study of the End Gas Autoignition and Shock Development by Flame Front and Local Hot Spot," ICDERS 2017, 1021, 2017.

22. Pal, P., Valorani, M., Arias, P.G., Im, H.G. et al., "Computational characterization of ignition regimes in a syngas/air mixture with temperature fluctuations," Proceedings of the Combustion Institute, 36(3), 3705-3716, 2017, doi: 10.1016/j.proci.2016.07.059.

23. Pal, P., Mansfield, A.B., Arias, P.G., Wooldridge, M.S. et al., "A computational study of syngas auto-ignition characteristics at highpressure and low-temperature conditions with thermal inhomogeneities," Combustion Theory and Modelling, 19(5), 587601, 2015, doi: 10.1080/13647830.2015.1068373.

\section{Contact Information}

Mohammed Jaasim Mubarak Ali

PhD Student

King Abdullah University of Science and Technology

Thuwal, Saudi Arabia, Ph: +966 565458 352,

Email: mohammedjaasim.mubarakali@kaust.edu.sa

\section{Acknowledgments}

This work was funded by King Abdullah University of Science and Technology (KAUST) and the computations utilized the KAUST supercomputing facility. The authors thank convergent science for providing the licenses for the code.

\section{Definitions/Abbreviations}

DDT Deflagration to Detonation

HRR Heat Release Rate

CA50 Crank angle 50 


$\begin{array}{ll}\phi_{0} & \text { Initial mean equivalence ratio } \\ T_{0} & \text { Initial mean temperature } \\ \mathrm{Sa} & \text { Sankaran number } \\ \mathrm{PDF} & \text { Probability Density Function } \\ \partial \tau_{\mathrm{i}} / \partial T & \text { Ignition delay gradient with respect to temperature } \\ \partial \tau_{\mathrm{ig}} / \partial \phi & \text { Ignition delay gradient with respect to equivalence ratio } \\ \beta & \text { Weight factor } \\ K & \text { K-factor } \\ T^{\prime} & \text { Root mean square of temperature } \\ \phi^{\prime} & \text { Root mean square of equivalence ratio } \\ u^{\prime} & \text { Root mean square of velocity } \\ P_{\mathrm{D}} & \text { Detonation probability normalized by sound speed } \\ S_{l} & \text { Laminar flame speed } \\ S_{\mathrm{sp}} & \text { Spontaneous front speed } \\ D \mathrm{a}_{l} & \text { Damköhler number } \\ \tau_{f} & \text { Flame time scale } \\ l_{e} & \text { Integral length scale } \\ \tau_{i g} & \text { Ignition delay time } \\ a & \text { Speed of sound } \\ P_{\mathrm{s}} & \text { Detonation probability normalized by Laminar } \\ & \text { flame speed }\end{array}$

Page 7 of 7 